\title{
The effect of requesting a reason for non-adherence to a guideline in a long running automated reminder system for PONV prophylaxis
}

Fabian O. Kooij ${ }^{1}$; Toni Klok²; Benedikt Preckel'; Markus W. Hollmann'; Jasper E. Kal²

${ }^{1}$ Academic Medical Centre, Dpt. Anaesthesia, Amsterdam, The Netherlands;

${ }^{2}$ OLVG hospital, Dpt. Anaesthesia, Amsterdam, The Netherlands

\section{Keywords}

Automated reminders, decision support, PONV

\section{Summary}

Background: Automated reminders are employed frequently to improve guideline adherence, but limitations of automated reminders are becoming more apparent. We studied the reasons for nonadherence in the setting of automated reminders to test the hypothesis that a separate request for a reason in itself may further improve guideline adherence.

Methods: In a previously implemented automated reminder system on prophylaxis for postoperative nausea and vomiting (PONV), we included additional automated reminders requesting a reason for non-adherence. We recorded these reasons in the pre-operative screening clinic, the OR and the PACU. We compared adherence to our PONV guideline in two study groups with a historical control group.

Results: Guideline adherence on prescribing and administering PONV prophylaxis (dexamethasone and granisetron) all improved compared to the historical control group (89 vs. 82\% ( $p<0.0001), 96$ vs $95 \%$ (not significant) and 90 vs $82 \%(p<0.0001))$ while decreasing unwarranted prescription for PONV prophylaxis (10 vs. $13 \%$ ). In the pre-operative screening clinic, the main reason for not prescribing PONV prophylaxis was disagreement with the risk estimate by the decision support system. In the OR/PACU, the main reasons for not administering PONV prophylaxis were: 'unintended non-adherence' and 'failure to document'.

Conclusions: In this study requesting a reason for non-adherence is associated with improved guideline adherence. The effect seems to depend on the underlying reason for non-adherence. It also illustrates the importance of human factors principles in the design of decision support. Some reasons for non-adherence may not be influenced by automated reminders.

\section{Correspondence to:}

Fabian 0. Kooij

Academic Medical Centre, University of Amsterdam

Department of anaesthesia

PO Box 22660,

1100 DD Amsterdam

Phone: +31205662533

Fax: +31206979441

Email: F.O.Kooij@amc.nl
Appl Clin Inform 2017; 8: 313-321

https://doi.org/10.4338/ACI-2016-08-RA-0138

received: August 15, 2016

accepted: January 19, 2017

published: March 29, 2017

Citation: Kooij FO, Klok T, Preckel B, Hollmann MW, Kal JE. The effect of requesting a reason for non-adherence to a guideline in a long running automated reminder system for PONV prophylaxis. Appl Clin Inform 2017; 8: 313-321

https://doi.org/10.4338/ACl-2016-08-RA-0138 


\section{Background and significance}

In recent years, automated reminders and electronic decision support have been used to improve behaviour of medical personnel, improve guideline adherence, documentation, billing and patient outcome [1-5]. Recently the effects of "alert fatigue" and unintended effects of decision support have also gained attention [6-8].

The expected effect of a decision support system depends on a multitude of factors, including the underlying guideline itself, as well as the design of the system, and the situation in which the decision support system is activated. From previous studies, automated reminders offering a recommendation instead of observation and requesting a reason from the user for not following recommendations offered by decision support systems, have all been suggested as features that may increase the effect of decision support systems [9]. From studies on human factors, the type, the design, the message and the timing of automated reminders also have been shown to influence their effect [10-12]. From a user acceptance point of view, it is also known that the system should be designed so that at least $80 \%$ of the reminders offer a correct observation and/or advice for optimal effectiveness [13].

In previous studies that evaluated the effects of decision support for prescribing and administering prophylactic medication for postoperative nausea and vomiting (PONV), we demonstrated that, although significantly improving guideline adherence, there was still residual non-adherence between 5 and 27 percent $[14,15]$. Although 100\% adherence is not realistic or even opportune for most guidelines, the particular guideline that was the basis for this decision support system had been written with the involvement and consent of all anesthesiologists and is to be applied to every single patient receiving anesthesia care in our hospital. Given the properties described above, it is clear to us that at $27 \%$ non-adherence the system was not working optimally and needed further improvement [14]. We reviewed the literature to identify potential improvements in the design of our decision support [9-12]. We modified our existing decision support system to include documentation of a reason for non-adherence when applicable.

\section{Objectives}

Since requesting a reason has previously been shown to improve guideline adherence, we hypothesized that this modification improves adherence to the local PONV prophylaxis guideline [9]. Moreover, on a more operational level, we intended to determine the underlying reasons for non-adherence in order to identify potential sources for improvement of our decision support systems.

\section{Methods}

This study was conducted in a 550-bed teaching hospital. An anaesthesia information management system (AIMS) workstation is present in all pre-operative screening offices, all 12 ORs and adjacent to all 15 PACU beds. Within the AIMS, a comprehensive decision support system on prescription and administration of PONV prophylaxis is implemented. PONV prophylaxis was to be prescribed to every patient with at least three risk factors on an Apfel's simplified risk score and consisted of dexamethasone $4 \mathrm{mg}$ iv. after induction of, and granisetron $1 \mathrm{mg}$ iv. before emergence from anaesthesia $[14,16]$.

In the pre-operative screening department, our decision support system consisted of an automated reminder based on Apfel's simplified risk score $[14,16]$. The system calculated the risk score for every patient and reminded the anaesthesiologist of the indication for PONV prophylaxis. A reminder message would be triggered if: (1) three or four risk factors were positive; (2) general anaesthesia was scheduled; and (3) no PONV prophylaxis had been prescribed. This message appeared immediately after selecting 'general anaesthesia' as the preferred anaesthesia technique. The system prompted the user for a response to the message (agree or disagree with the recommendation) and automatically prescribed PONV prophylaxis if the user agreed. Since our AIMS is not a Computerized Physician Order Entry (CPOE) system, there were no specific medications prescribed at that 
point in time, just an order for "PONV prophylaxis according to protocol". For the purpose of this study, a second reminder appeared immediately if a negative response to the first reminder was entered. This reminder requested the reason for not prescribing PONV prophylaxis.

There were two different reminders in the OR/PACU suggesting administering of PONV prophylaxis to the clinicians [15]. The first reminder was programmed to appear 10 minutes after induction of anaesthesia (OR reminder). The second reminder was programmed to occur upon arrival in the PACU (PACU reminder). The OR reminder showed a message if: (1) PONV prophylaxis had been prescribed; (2) general anaesthesia had been recorded; and (3) dexamethasone (or other steroids) had not been recorded as 'administered'. If dexamethasone (or other steroids) had not been administered another 10 minutes later, a second reminder appeared requesting a reason for non-adherence. This reminder kept re-appearing every 10 minutes until either a reason was documented or steroids were administered.

The PACU reminder checked patient data upon arrival in the PACU and was triggered if: (1) PONV prophylaxis had been prescribed; (2) general anaesthesia had been given; and (3) no granisetron had been recorded as 'administered' yet. Again, a second reminder requesting a reason appeared 10 minutes later if granisetron had still not been administered.

For all of the reminders, a response was required to continue working with the AIMS. However, the response required was one mouse-click or keystroke, validating only that the user had seen the message. The users (anaesthesiologists, anaesthesia residents and anaesthesia nurses) were never forced to adhere to the protocol; they were only repeatedly reminded of it.

As described, the second tier of reminders requested a reason for non-adherence. Based on known reasons for non-adherence, as well as personal experience and conversations with colleagues, we predefined several choices which we expected to cover most of the reasons for non-adherence to the departmental protocol [17]. The predefined reasons were: mistake/forgotten, disagree with guideline, disagree with risk estimate, contraindication, and documentation error (OR/PACU only). To facilitate any other possible reason, an option "other" and a free text field were also available.

In this observational study we report three different patient sets: a first study group in the pre-operative screening department, a second study group in the OR/PACU, and a historical control group. In both study groups, the second tier of reminders requesting a reason for non-adherence was employed. The division between pre-operative screening and OR/PACU within the study groups was made because the date of pre-operative screening can be up to 6 months prior to surgery, a different anaesthesiologist may be involved, and the two visits (pre-operative and OR) should be considered unrelated within the scope of this study. For example, one patient may have been pre-operatively screened before the study period and may have received anaesthesia within the study period. The study groups included all consecutive adult patients for elective, non-cardiac surgery in our hospital. The need for informed consent as well as full IRB approval was waived by the institutional IRB (Medisch Ethische Commissie, OLVG hospital). Data collection was done automatically within the AIMS database and data were extracted from the AIMS database after study completion. For this observational study, we arbitrarily decided to set the sampling time frame to 9 months (or approximately 10000 non-cardiac patients) for the OR/PACU group and 2 years (or approximately 20000 patients) for the pre-operative screening group. For the historical control group, we used a set of patients from a previous study, which was treated similarly using the same automated reminders with the exception of the request for a reason for non-adherence in the second tier of reminders [15].

For comparison of the different study groups, proportions were calculated. Statistical significance of differences was assessed by Chi square statistic using SPSS (version 19).

\section{Results}

In the pre-operative screening department, 27332 patients were screened while the additional reminders requesting the reason for non-adherence were active (between Jan $1^{\text {st }} 2009-\operatorname{Dec} 31^{\text {st }} 2010$ ). In the OR, there were 11270 patients who received anaesthesia while the additional reminders were active in the OR/PACU (between Jan $1^{\text {st }} 2009$ - Oct 11 $1^{\text {th }}$ 2009). In the historical control group (Jan $18^{\text {th }} 2006-$ April $\left.30^{\text {th }} 2006\right), 2594$ patients were screened and/or received anaesthesia. Demographic properties of the populations are shown in Table 1 . 
Table 2 demonstrates that guideline adherence was better in the study groups when compared to the historical control group. Prophylaxis was prescribed to high-risk patients more often in the study group ( $89 \%$ vs $82 \%, \mathrm{p}<0.0001$ ), while simultaneously prescription to 'low risk' patients decreased compared to the control group ( $10 \%$ vs $13 \%, \mathrm{p}<0.0001)$.

In the OR, administration of dexamethasone remained the same (95 vs $96 \%$, not significant) while administration of granisetron further improved ( 82 vs 90\%, p < 0.0001). Administration of both dexamethasone and granisetron to low risk patient remained the same.

Although the guideline adherence improved, in the study group still 496 patients (11\% of high risk patients with scheduled general anaesthesia) were not prescribed PONV prophylaxis initially. In 97 of these patients, a reason for non-adherence was documented. Subjective disagreement with the risk estimate by the decision support system was the main reason for not prescribing PONV prophylaxis (44 times). In a small proportion of patients (31 out of 496 patients, 6\%), the reason request caused a change in policy, resulting in either prescribing PONV prophylaxis after all (21 patients), or changing the planned anaesthesia technique to regional instead of general anaesthesia (10 patients).

In the OR/PACU, the main reasons for not administering dexamethasone and granisetron were: unintended non-adherence (33\% for dexamethasone and $42 \%$ for granisetron, respectively), and failure to document the administration of medication ( $26 \%$ and $44 \%$, respectively). A change of policy was more frequent than in the pre-operative screening clinic. An additional 65 out of 131 patients (50\%) received dexamethasone after the reason request, and 290 out of 452 patients (64\%) received granisetron after the second reminder with the reason request.

\section{Discussion}

This observational study demonstrated that requesting a reason for non-adherence to the local PONV prophylaxis guideline was associated with an increase in PONV prophylaxis prescription and administration without causing an increase in inappropriate PONV prophylaxis prescription and administration to low risk patients. In addition, we gained interesting insights in some of the reasons for residual non-adherence in a long running clinical decision support system.

Although not scientifically reported, it is a known phenomenon that users will in some situations discard a reminder by routinely clicking the upper or lowermost option available in a reminder or just press enter to select the default option (if available). Although we had no formal process of eliminating that this happened, we do not feel this played a big role. First of all, no default reason was pre-selected so just pressing enter would not select any reason. Secondly, there was a marked difference between the pre-operative screening department and the OR/PACU in the most frequently selected reasons for non-adherence. The most frequent reason for non-adherence at the pre-operative screening department was disagreement with the guideline (the third option from the top). In the OR, failure to document (second option from the top), and unintended non-adherence (first option from the top) were the two most frequent explanations for not following the suggestion by the decision support system. Since the predefined reasons were sorted in the same order in the pre-operative screening clinic as in the OR/PACU, this would make "routine selection" less likely.

Moreover, there was a marked difference in the effect on adherence of the reason request; in the pre-op screening clinic, only $4 \%$ of reminders triggered a prescription. In the OR and PACU, 50 $64 \%$ of reason requests triggered additional administration of medication.

Looking at the medical evidence supporting our PONV guideline and decision support system, the risk scoring was based on publications by Apfel et al., while the choice of drugs (dexamethasone and a 5-HT-3 receptor antagonist) was made on the basis of the available Cochrane review on drugs for PONV prevention and therapy $[16,18,19]$. Reviewing this evidence, we concluded that the decision support rules implemented were evidence based and that our guideline most likely did not overestimate PONV risk. In contrast, our guideline and decision support system may have slightly underestimated the risk in medium-risk patients ( 1 or 2 positive risk factors) [20].

Since the design of a decision support system is crucial to its efficacy, we evaluated the design of our automated reminders $[9,12]$. The review by Kawamoto et al. suggests a number of properties that may predict the effectiveness of decision support systems [9]. Our decision support system possesses all independent predictors for effectiveness as defined by this review: automated activation 
within workflow, specific actionable advice, requesting a reason, and being computer based. Another review by Phansalkar et al. evaluated human factors principles and their application to automated reminders [12]. Again, most of these principles were incorporated in our decision support system.

Another human factor principle that may be important is workload. It is well known that performance can decrease as a result of distractions by increased workload. This factor may have influenced the present study. The time frame in which granisetron is administered and documented, between the end of surgery and the arrival in the PACU, is a high workload situation and therefore particularly susceptible to distractions resulting in decreased performance and documentation failure. This may explain the relatively large effect of automated reminders on adherence in this situation. In the design of our decision support system, we have chosen not to interfere with busy clinical care during emergence from anaesthesia and transport to the PACU, but to remind the user of the omission upon arrival in the PACU [15]. This illustrates the importance of timing of automated reminders.

The request for a reason itself also seems to have an effect on residual non-adherence. More specifically, the second reminder (requesting a reason) has led to the administration of the PONV medication in $50-64 \%$ of times it was shown (an extra $3-17 \%$ of all high-risk patients receiving general anaesthesia).

Besides the possible beneficial effect of the reason request on residual non-adherence, another finding is that residual non-adherence may have decreased considerably over time. The difference is most clear-cut in the outpatient clinic for prescription of PONV prophylaxis. In the study described in our original report, the non-adherence for prescribing PONV prophylaxis decreased from $63 \%$ to $27 \%$ using reminders [14]. This decreased even further to $18 \%$ in the intervention group of our second study [15]. During the current study period, three years later, this was only $11 \%$. This finding is in contradiction with some studies done in CPOE systems, where reminders are actually overridden more frequently over time [8]. The difference between the CPOE literature and our study may be in the relative frequency of overrides. In the report by Topaz, override frequencies of up to $89 \%$ are reported [8]. This is quite high in comparison to the overrides in our system (between $4 \%$ and $18 \%$, depending on the specific reminder). So, placing our results in context of this report, although we demonstrated absence of a short term learning effect (weeks) in our original report, we now think there may be a long term "learning" effect (years) [14]. This may be due to a combination of factors, including but not limited to continuous education and feedback on guideline adherence by the reminders, increased confidence in the suggestions made by the decision support system, and a change in perceived importance of PONV prophylaxis over time.

\section{Limitations}

The most important limitation of this study is that only associative conclusions can be drawn. Due to the case-control type design, there is no possibility to conclude on causation. Moreover, due to the longer period of time between the control group and the intervention group, it is possible that the perception regarding the guideline by providers had changed in between the two study periods. Also, the sample size of the control group is rather small in comparison to the intervention groups. Nevertheless, we feel the study gives an interesting insight in both the potential effects of requesting a reason for non-adherence to an automated reminder as well as the actual reasons for non-adherence. Finally, for a complete evaluation of the system, we could have sought user feedback and try to gain insight in the experience, for example by using a survey.

\section{Conclusion}

In conclusion, the request for a reason for non-adherence was in itself associated with an increase in guideline adherence in a long running automated reminder system for PONV prophylaxis. Moreover, we have identified different reasons for non-adherence in the setting of automated decision support. The present study illustrates that human factors are important to account for when design- 
ing decision support systems. In addition, not all reasons for non-adherence may be amenable by decision support.

\section{Multiple Choice questions}

1. The reason for non-adherence to a guideline which is most amenable by automated reminders is:

A) Disagreement with the contents of the guideline

B) Unintentional non-adherence

C) Unavailability of the medication suggested

D)Contra-indication for the medication suggested

Unintentional non-adherence, where the user would have followed the guideline if he had known about the guideline and had realized it was applicable to this patient, is among the most amenable reasons for non-adherence. Showing the reminder brings to the attention that the guideline exists and is applicable to this patient.

For optimal efficacy, the reminder is shown at the right time and place, and shows an actionable advice for the user to actually perform. This is demonstrated by the significant improvement of adherence by both our original reminder system and again by the additional reminder in the OR/ PACU requesting a reason for non-adherence up to that moment. The most frequent reasons documented were unintentional non-adherence.

Disagreement is most likely not amenable by automated reminders only, as demonstrated by the almost complete absence of change in the pre-operative screening department.

Unavailability of medication and contra-indication for medication are off course not amenable by automated reminders.

2. The following factors are important for optimal performance of a clinical decision support system:

E) The vendor of the system the reminders are embedded in

F) Timing and of reminders in relation to workload

$\mathrm{G})$ Eliminating all false positive reminders

$\mathrm{H})$ Switching the reminders on and off periodically

An important factor in the efficacy of a reminder system is the timing of the reminders. Showing a reminder at a time with high workload most likely makes this reminder less effective. This is illustrated by the PACU reminders: the additional adherence generated by the second reminder is the largest in the PACU group when compared to the other groups. Moreover, the reasons documented show the highest incidence of unintentional non-adherence as well as failed documentation.

Eliminating all false positive reminders is an important principle, but also utopia. Since an exception can be formulated for almost any guideline, the elimination of all false positive reminders is not realistically achievable.

Turning the reminders on and off periodically is probably not a good idea, since over the longer period we have had our reminders active we have seen a big initial increase in adherence, but also an ongoing continuous improvement of adherence.

\section{Clinical relevance statement}

This study provides clinicians insights into reasons for not following recommendations of an automated reminder system and provides information on potential optimization in design and timing as well as limitations of a decision support system.

\section{Conflict of interest}

Financial support for this study was provided by the authors' employing institutions only. The funding agreement ensured the authors' independence in designing the study, interpreting the data, writing, and publishing the report.

\section{Human subjects}

No human subjects were involved in the project. 
Table 1 This table shows basic demographics for all three study periods. No statistical comparison between the groups was done.

\begin{tabular}{|l|c|c|c|}
\hline & Control & \multicolumn{2}{l|}{ Study population } \\
\cline { 2 - 4 } & & Pre-Operative & OR/PACU \\
\hline & N (\%) & N (\%) & N (\%) \\
\hline Total number of patients & 2594 & 27332 & 11270 \\
\hline ASA class & & & $6300(56)$ \\
\hline I & $1520(59)$ & $15053(55)$ & $3517(32)$ \\
\hline II & $968(37)$ & $8594(31)$ & $965(9)$ \\
\hline III & $102(4)$ & $2463(9)$ & $153(1)$ \\
\hline IV & $3(0,1)$ & $396(2)$ & $18(0,2)$ \\
\hline V & $0(0)$ & $37(0,1)$ & $6898(61)$ \\
\hline Planned general anaesthesia & $1729(67)$ & $16052(59)$ & $7472(66)$ \\
\hline Executed general anaesthesia & $1768(68)$ & Unknown & \\
\hline PONV risk factors & & & $6454(57)$ \\
\hline Female gender & $1489(57)$ & $15771(58)$ & $6501(57)$ \\
\hline Non smoker & $1586(61)$ & $15197(55)$ & $2138(19)$ \\
\hline Previous PONV or motion sickness & $614(24)$ & $5249(19)$ & $5041(45)$ \\
\hline Expected opioid use & $1264(49)$ & $11662(42)$ & $2934(26)$ \\
\hline High-risk patients (3 or more risk factors) & $781(30)$ & $6881(25)$ & \\
\hline
\end{tabular}

Table 2 Changes in guideline adherence between the control period and the intervention period. A statistically significant increase in prescription and administration of PONV prophylaxis to high-risk patients was observed. Prescription and administration to low-risk patients remained the same or decreased.

\begin{tabular}{|l|c|c|l|}
\hline & $\begin{array}{l}\text { Control } \\
\text { N (\%) }\end{array}$ & $\begin{array}{l}\text { Study } \\
\text { N (\%) }\end{array}$ & \\
\hline Pre-operative: PONV prophylaxis prescription & & & \\
\hline High-risk patients with scheduled G.A. & $523(100)$ & $4522(100)$ & \\
\hline PONV prophylaxis prescribed & $426(82)$ & $4026(89)$ & $\mathrm{p}<0.0001$ \\
\hline Low-risk patients with scheduled G.A. & $1206(100)$ & $11530(100)$ & \\
\hline PONV prophylaxis prescribed & $161(13)$ & $1115(10)$ & P $<0.0001$ \\
\hline OR/PACU: PONV prophylaxis administration & & & \\
\hline High-risk patients with received G.A. & $541(100)$ & $1958(100)$ & \\
\hline Prophylaxis prescribed & $433(80)$ & $1642(84)$ & P $=0,03$ \\
\hline Dexamethasone administered* & $411(76 / 95)$ & $1576(80 / 96)$ & n.s. \\
\hline Granisetron administered* & $356(66 / 82)$ & $1480(76 / 90)$ & P $<0.0001$ \\
\hline Low-risk patients with received G.A. & $1227(100)$ & $5514(100)$ & \\
\hline Prophylaxis prescribed & $158(13)$ & $457(8)$ & P $<0.0001$ \\
\hline Dexamethasone administered & $181(15)$ & $871(16)$ & n.s. \\
\hline Granisetron administered & $176(14)$ & $805(15)$ & n.s. \\
\hline
\end{tabular}

G.A. general anaesthesia

n.s. not significant

${ }^{*} \mathrm{~N}$ (\% of high risk patients with G.A. / \% of high risk patients with G.A. and prescription) 
Table 3 Reasons for non-adherence in the high-risk study population. For the prescription of prophylaxis, disagreement with the risk estimate was the most frequently entered reason. In the OR and PACU, unintended non-adherence (forgotten/mistake or failure to document) was the reason most frequently documented.

\begin{tabular}{|l|c|c|c|}
\hline & Prescribing & \multicolumn{1}{l}{ Administration } \\
\cline { 2 - 4 } & & Nexamethasone & Granisetron \\
\cline { 2 - 5 } & N (\%) & N (\%) & N (\%) \\
\hline PONV prophylaxis NOT prescr./admin. & $496(100)$ & $131(100)$ & $452(100)$ \\
\hline Reason documented & $97(20)$ & $131(100)$ & $252(100)$ \\
\hline Prescr./admin. after reason request & $21(4)$ & $65(50)$ & NA \\
\hline Change of technique to regional & $10(2)$ & NA & $452(100)$ \\
\hline Total reasons documented & $97(100)$ & $131(100)$ & $188(42)$ \\
\hline Unintended (forgotten / by mistake) & $19(20)$ & $43(33)$ & $198(44)$ \\
\hline Administered but not registered & NA & $34(26)$ & $46(10)$ \\
\hline Guideline overestimates risk & $44(45)$ & $25(20)$ & $3(1)$ \\
\hline Contraindication & $16(17)$ & $2(2)$ & NA \\
\hline ICU patient/other steroid given* & $5(5)$ & $7(5)$ & $17(4)$ \\
\hline Other & $13(13)$ & $20(15)$ & \\
\hline
\end{tabular}

NA: Not applicable

* This reason was entered using the free text option by the users and categorized by the authors afterwards. 


\section{References}

1. Dexter PR, Perkins S, Overhage JM, Maharry K, Kohler RB, McDonald CJ. A computerized reminder system to increase the use of preventive care for hospitalized patients. N Engl J Med 2001;345(13): 965-970.

2. O'Reilly M, Talsma A, VanRiper S, Kheterpal S, Burney R. An anesthesia information system designed to provide physician-specific feedback improves timely administration of prophylactic antibiotics. Anesth Analg 2006; 103(4): 908-912.

3. Rood E, Bosman RJ, van der Spoel JI, Taylor P, Zandstra DF. Use of a computerized guideline for glucose regulation in the intensive care unit improved both guideline adherence and glucose regulation. J Am Med Inform Assoc 2005; 12(2): 172-180.

4. Spring SF, Sandberg WS, Anupama S, Walsh JL, Driscoll WD, Raines DE. Automated Documentation Error Detection and Notification Improves Anesthesia Billing Performance. Anesthesiology 2007; 106(1): 157-163.

5. Kooij FO, Vos N, Siebenga P, Klok T, Hollmann MW, Kal JE. Automated reminders decrease postoperative nausea and vomiting incidence in a general surgical population. BrJAnaesth 2012; 108(6): 961-965.

6. Khalifa M, Zabani I. Improving Utilization of Clinical Decision Support Systems by Reducing Alert Fatigue: Strategies and Recommendations. Studies in health technology and informatics 2016; 226: 51-54.

7. Epstein RH. Postoperative nausea and vomiting, decision support, and regulatory oversight. Anesth Analg 2010; 111(2): 270-271.

8. Topaz M, Seger DL, Slight SP, Goss F, Lai K, Wickner PG, Blumenthal K, Dhopeshwarkar N, Chang F, Bates DW, Zhou L. Rising drug allergy alert overrides in electronic health records: an observational retrospective study of a decade of experience. Journal of the American Medical Informatics Association: JAMIA 2016; 23(3): 601-608.

9. Kawamoto K, Houlihan CA, Balas EA, Lobach DF. Improving clinical practice using clinical decision support systems: a systematic review of trials to identify features critical to success. BMJ 2005; 330(7494): 765.

10. Jung M, Riedmann D, Hackl WO, Hoerbst A, Jaspers MW, Ferret L, Lawton K, Ammenwerth E. Physicians' perceptions on the usefulness of contextual information for prioritizing and presenting alerts in Computerized Physician Order Entry systems. BMC Med Inform Decis Mak 2012; 12: 111.

11.van der Sijs H, Baboe I, Phansalkar S. Human factors considerations for contraindication alerts. Stud Health Technol Inform 2013; 192: 132-136.

12.Phansalkar S, Edworthy J, Hellier E, Seger DL, Schedlbauer A, Avery AJ, Bates DW. A review of human factors principles for the design and implementation of medication safety alerts in clinical information systems. J Am Med Inform Assoc 2010; 17(5): 493-501.

13. Wickens CD, Dixon SR. The benefits of imperfect diagnostic automation: a synthesis of the literature. Theoretical Issues in Ergonomics Science 2007; 8(3): 201-212.

14. Kooij FO, Klok T, Hollmann MW, Kal JE. Decision support increases guideline adherence for prescribing postoperative nausea and vomiting prophylaxis. AnesthAnalg 2008; 106(3): 893-898.

15. Kooij FO, Klok T, Hollmann MW, Kal JE. Automated reminders increase adherence to guidelines for administration of prophylaxis for postoperative nausea and vomiting. EurJAnaesthesiol 2010; 27(2): 187-191.

16. Apfel CC, Laara E, Koivuranta M, Greim CA, Roewer N. A simplified risk score for predicting postoperative nausea and vomiting: conclusions from cross-validations between two centers. Anesthesiology 1999; 91(3): 693-700.

17. Cabana MD, Rand CS, Powe NR, Wu AW, Wilson MH, Abboud PA, Rubin HR. Why don't physicians follow clinical practice guidelines? A framework for improvement. JAMA 1999; 20; 282(15): 1458-1465.

18. Apfel CC, Kranke P, Eberhart LH, Roos A, Roewer N. Comparison of predictive models for postoperative nausea and vomiting. Br J Anaesth 2002; 88(2): 234-240.

19. Carlisle JB, Stevenson CA. Drugs for preventing postoperative nausea and vomiting. Cochrane Database Syst Rev 2006; 3: CD004125.

20. Gan TJ, Meyer TA, Apfel CC, Chung F, Davis PJ, Habib AS, Hooper VD, Kovac AL, Kranke P, Myles P, Philip BK, Samsa G, Sessler DI, Temo J, Tramer MR, Vander KC, Watcha M. Society for Ambulatory Anesthesia guidelines for the management of postoperative nausea and vomiting. Anesth Analg 2007; 105(6): 1615-1628. 